\title{
Efecto de las estrategias competitivas y los recursos y capacidades orientados al mercado sobre el crecimiento de las organizaciones
}

\section{Carmen Berenice}

Ynzunza Cortés

Universidad Tecnológica de Querétaro

bynzunza@uteq.edu.mx

\section{Juan Manuel}

Izar Landeta

Universidad Autónoma

de San Luis Potosí

jmizar@uaslp.mx

\section{Resumen}

Este estudio analiza las relaciones entre las fuerzas de mercado, las estrategias competitivas, los recursos y capacidades orientados al mercado, así como el desempeño organizacional. La investigación se realizó en Pyme del sector industrial del estado de Querétaro, con un muestreo de conveniencia no probabilístico; asimismo, se diseñó un modelo estructural para probar las relaciones entre las variables, las cuales se analizaron con el software AMOS. El tamaño de muestra alcanzado fue de 116 y la recopilación de información se hizo mediante entrevistas. Las fuerzas de mercado, la estrategia competitiva y la orientación a mercado fueron medidas con las escalas de Kohly y Javorsky (1990), Segev (1987) y Narver y Slater (1990). Los ítems para el desempeño organizacional y los recursos de tecnología e innovación fueron diseñados. Los hallazgos prueban que las fuerzas de mercado tienen un impacto grande en la estrategia competitiva, el cual no es significativo sobre los recursos y capacidades, y es negativo respecto al desempeño, medido por el crecimiento organizacional. El efecto de la estrategia competitiva ha sido significativo sobre los recursos y capacidades de orientación a mercado, tecnología e innovación y no significativo respecto al desempeño. Estos recursos y capacidades influyen de manera significativa en el desempeño organizacional. Los resultados de este estudio enfatizan la implementación de estrategias prospectoras y analizadoras, que crean recursos y capacidades que den valor, con beneficios económicos para las empresas, así como los de orientación a mercado, tecnología e innovación. Estos hallazgos son consistentes con los encontrados en otras investigaciones realizadas en otros países.

Palabras clave: estrategia, mercado, recursos, capacidades, desempeño. 


\title{
Effect of competitive strategies and market oriented resources and capabili- ties on organizational growth
}

\begin{abstract}
This study analyzes the relationships between market forces, competitive strategies, market orientation, innovation, technological resources and capabilities, and organizational performance. The study was made in small firms (SMEs) in the industrial sector in the State of Queretaro with a non-probabilistic convenience sampling. A structural model was designed to test the variable relationships, using Amos software. The sample size was 116 and interviews were applied to collect data. To measure market forces, competitive strategies and market orientation Kohli and Jaworsky (1990), Segev (1987) and Narver and Slater (1990) scales were used. The items to measure technological and innovation capabilities and organizational performance were designed. The results of the study show that market forces have a significant impact on the competitive strategy, a non-significant impact on resources and capabilities and a negative one on performance, this last one being measured by organizational growth. The effect of competitive strategy is significant on market orientation, technological and innovation resources and capabilities and non-significant on organizational performance. These resources and capabilities have a significant influence on organizational performance. The results of this research emphasize the implementation of prospecting and analyzing strategies that create resources and capabilities which give value such as market orientation, technology and innovation, with economical benefits for organizations.
\end{abstract}

Keywords: strategy, market, resources, capabilities, performance.

\section{Introducción}

Para crear crecimiento y generar un desempeño sostenido en el largo plazo, en un ambiente dinámico y cambiante, donde la globalización, el incremento de la competencia, las nuevas tecnologías y las demandas de los consumidores por nuevos productos son una constante, las organizaciones requieren alinearse a las oportunidades y amenazas externas de la industria e implementar una estrategia que les permita generar los recursos y capacidades organizacionales que les brinden una ventaja competitiva.

La globalización, al igual que los avances tecnológicos y un entorno de rápidos cambios, generan asimismo oportunidades tanto para organizaciones grandes 
como pequeñas. Sin embargo, son éstas últimas las que tienen más posibilidad, por su flexibilidad, de adaptarse y responder al ambiente en el que operan (Freeman et al., 1982); su capacidad de innovación es mayor, aun cuando su proceso de innovación sea más lento, debido a la problemática que las caracteriza. Las Pyme son un elemento clave para la competitividad y el crecimiento; un motor primario de la innovación en los países en desarrollo.

En México, las Pyme tienen una función fundamental en la industria y la economía, pues representan más del $99.8 \%$ de las empresas establecidas, emplean una fuerza laboral del $78.5 \%$ y aportan el $52 \%$ del producto interno bruto nacional (INEGI, 2004). Sin embargo, la apertura de mercados y el proceso de transformación de la industria trajo no sólo inversión y creación de empleos, sino también un incremento importante en el número de competidores provenientes de otros países, con procesos innovadores de manufactura, tecnología, mercadotecnia y dirección, que garantizan mayores posibilidades de éxito, situación que ha repercutido fuertemente en el progreso de las Pyme.

Pocos empresarios mexicanos están conscientes de la importancia de desarrollar herramientas estratégicas competitivas que les permitan adaptarse rápidamente a las condiciones del mercado y mantener una posición competitiva, por lo cual todavía muchas firmas mantienen posiciones defensivas o reactivas que les impiden alcanzar las condiciones tecnológicas, económicas, competitivas y de mercado que favorezcan su desarrollo y crecimiento.

Las firmas que establecen una clara estrategia alcanzan un mejor desempeño; los recursos y capacidades internos son elementos críticos para generar una ventaja competitiva (Barney, 1991; Grant, 1991) y los recursos y capacidades de orientación a mercado propician los comportamientos necesarios para mejorar las capacidades internas que potencializan el desarrollo de nuevos productos, crean valor superior a los clientes y, así, un desempeño superior continuo para el negocio (Narver y Slater, 1990). Además, son un factor necesario e importante para constituir un ambiente óptimo vinculado con los recursos y capacidades de tecnología e innovación, por lo que - al considerar ésta un área de interés que puede generar información importante para el desempeño de las organizaciones y ser un aporte al desarrollo del conocimiento de las mismas- este estudio busca explorar el vínculo existente entre la estrategia competitiva, los recursos y capacidades orientados al mercado, el desempeño organizacional y las relaciones existentes entre ellos. 
El conocimiento y divulgación de estrategias utilizadas en otras organizaciones que han probado ser exitosas puede contribuir a acelerar el crecimiento de otras empresas que convergen en un ambiente competitivo, en clara desventaja respecto a competidores y grandes empresas que se han instalado en el país. Las preguntas e hipótesis de investigación planteadas para realizar este estudio se detallan a continuación.

\section{Preguntas de investigación}

¿Qué relación existe entre las fuerzas de mercado y las estrategias competitivas orientadas a mercado? ¿Cuál es el impacto de las fuerzas de mercado, la estrategia competitiva y los recursos y capacidades orientados al mercado sobre el crecimiento organizacional? ¿Cuál es el efecto de las estrategias competitivas sobre el desarrollo de recursos y capacidades de orientación a mercado y tecnología e innovación?

\section{Hipótesis de investigación}

$\mathrm{H}_{1}$. Existe una relación significativa entre las fuerzas de mercado y la implementación de estrategias competitivas orientadas a mercado.

$\mathrm{H}_{2}$. Existe una relación significativa entre las fuerzas de mercado y el desarrollo de recursos y capacidades orientados a mercado.

$\mathrm{H}_{3}$. Existe una relación significativa entre las fuerzas de mercado y el crecimiento de las organizaciones.

$\mathrm{H}_{4}$. Existe una relación significativa entre la implementación de estrategias competitivas y el desarrollo de recursos y capacidades orientados a mercado.

$\mathrm{H}_{5}$. Existe una relación significativa entre el desarrollo de recursos y capacidades orientados a mercado y el crecimiento de las organizaciones.

$\mathrm{H}_{6}$. Existe una relación significativa entre la implementación de estrategias competitivas orientadas a mercado y el crecimiento de las organizaciones 


\section{Marco contextual}

\section{La pequeña y mediana empresa en México}

La contribución de las Pyme a la actividad económica del país se da no sólo en términos de unidades económicas, ocupación laboral y/o producción, sino también de desarrollo, ya que asumen un papel relevante en las economías regionales o locales. En cuanto a su situación —en términos generales- se visualizan como organizaciones con un bajo volumen de exportaciones, con una producción orientada al sector interno, con limitado acceso al sistema financiero formal, con una baja tasa de supervivencia, poco insertadas al proceso de globalización y con una alta dependencia a los factores económicos y de distribución del ingreso. Sin embargo, se les reconoce la flexibilidad de sus procesos productivos, la demanda especializada y la capacidad de integrarse a diversos esquemas de subcontratación y encadenamientos productivos, su enfoque hacia la diversificación y autonomía, así como el establecimiento de relaciones de interdependencia con las grandes empresas (Díaz, 2002; Kauffman, 2001).

Su desarrollo en el país se basa en gran medida en las políticas y programas gubernamentales de fomento y apoyo, las cuales se han enfocado a la adquisición de bienes de capital, a la integración de capital de trabajo, a la reestructura de las instalaciones, a la provisión de servicios de consultoría en organización, dirección y mercado; asimismo, en la conformación de la Subsecretaría para la Pequeña y Mediana Empresa y diversos organismos de apoyo que buscan insertar y posicionar exitosamente a este tipo de empresas en los mercados nacionales e internacionales con el fin de incrementar su contribución a la generación del empleo y el ingreso, lo que ha resultado en un notable crecimiento del sector.

\section{La pequeña y mediana empresa en Querétaro}

Querétaro, al igual que muchos otros estados del país, no está ajeno a los problemas asociados a la apertura de mercados, el incremento de la competencia, la demanda de innovaciones y el crecimiento en el uso de la tecnología. La instalación de un número significativo de empresas del exterior en las últimas décadas le ha traído desarrollo y bienestar económico. Sin embargo, no ha fortalecido ni detonado el crecimiento de las Pyme, pero sí ha propiciado su rezago paulatino. En esta entidad existen 61627 empresas, de las cuales el 99.43\% son Pyme que proveen el $63.3 \%$ del empleo total y aportan el $42.25 \%$ de la producción bruta. La 
estructura de negocio dominante es la de microempresario, con la mayor tasa de ocupación en la micro y gran empresa, y la producción total bruta en la grande y mediana empresa.

En cuanto a los sectores de actividad, el de manufactura representa el 10\%; sin embargo, en la producción total contribuye con el 57\% (INEGI, 2004). La manufactura industrial se concentra mayormente en la capital y los municipios aledaños $(88.27 \%)$. Las áreas con mayor participación son la producción metálica y de autopartes; química; caucho y plástico; eléctrica y electrónica; alimentos, bebidas y tabacos; y el resto se distribuye en las otras actividades económicas (SEDESU, 2006). La mayoría de las empresas instaladas (99.43\%) — después de varios años de exposición a los mercados internacionales y a las prácticas de grandes empresas nacionales y trasnacionales - siguen siendo micro, pequeñas y medianas empresas, predominando por supuesto las primeras. Las que presentan el mayor crecimiento son las del sector servicios (64.5\% anual); por su parte, el sector industrial ha crecido en promedio a una tasa del $1 \%$ anual, lo que es una clara evidencia de la etapa de estancamiento que ha estado viviendo el sector en los últimos años.

Las pequeñas y medianas empresas no han podido beneficiarse del conocimiento generado por estas grandes empresas. Si bien en el estado convergen una serie de factores que podrían potencializar su desarrollo (centros de investigación y desarrollo tecnológico, universidades e instituciones gubernamentales y financieras), pocas de las empresas locales poseen tecnología avanzada, puesto que la mayoría desarrollan más bien trabajos de maquila y enfrentan condiciones económicas, tecnológicas y de mercado adversas (Sverket et al., 2001), a la par de los factores endógenos y exógenos que son característicos de las mismas, que inciden en su desempeño y que son similares a los del resto de la industria del país.

La generación al valor de la producción, el número de unidades existentes y el personal ocupado de este sector son indicadores de la relevancia del mismo para la economía y el desarrollo del estado. Sin embargo, las Pyme no han alcanzado el grado de evolución necesario para transformarse en medianas y grandes empresas, por lo que aún permanecen, en porcentajes relativamente altos, como micro y pequeñas empresas. Poco se sabe respecto al potencial de éstas para cubrir los estándares de las grandes empresas nacionales y trasnacionales. No se conocen las capacidades organizacionales y tecnológicas que poseen y que pudiesen contribuir a mejorar su vínculo y a fortalecerlas, por lo que las instituciones de apoyo se ven 
limitadas para canalizar sus esfuerzos a la generación de los recursos necesarios para mejorar el crecimiento y la competitividad de este sector.

\section{Marco teórico}

Entender las fuentes de ventaja competitiva ha sido una de las áreas de investigación principales en la administración estratégica. Diversos estudios en distintas partes del mundo coinciden en que la base del éxito y la ventaja competitiva yace en la estrategia competitiva seleccionada, los recursos y capacidades que la firma posee y su capacidad de adaptación a las condiciones del mercado (Barney, 1991; Porter, 1980; Grant, 1991). La estrategia competitiva puede incentivar los aspectos que contribuyan en gran medida a determinar el desempeño, así como la innovación, el desarrollo y la capacidad tecnológica (Conan et al., 1990). Otros estudios también refieren que las organizaciones exitosas son aquellas que logran una posición competitiva, generan un enfoque sistemático de adaptación al medio ambiente (Porter,1980; Miles y Snow, 1978) y tienen conductas estratégicas proactivas integradas hacia la innovación, calidad y satisfacción del cliente (Camison, 1997), enfatizando que el ambiente tiene mucha más influencia en formar las estrategias de las firmas que ningún otra cosa, por lo cual sugieren que las compañías deberían poner más énfasis en adaptarse a su medio ambiente e incluso proponen orientaciones estratégicas generadoras de cambios, innovación y respuesta rápida a las condiciones ambientales, como las prospectoras y analizadoras (Miles y Snow, 1978). También señalan la existencia de una relación significativa entre este tipo de actividades estratégicas y la formulación de recursos y capacidades (Ynzunza, 2010).

En relación con el medio ambiente, la literatura tiene pocos estudios empíricos de los efectos de éste en las organizaciones. La mayoría se enfoca principalmente a explorar en qué grado las empresas están orientadas al mercado (Narver y Slater, 1990; Kohli y Jaworsky, 1990), pero poco se ha avanzado respecto a conocer sus consecuencias en las organizaciones. No obstante, algunos refieren dimensiones ambientales e incluso niveles de variación que requerirían el establecimiento de estrategias y desarrollo de recursos y capacidades determinadas (Aragón y Sharma, 2003), inclusive toda una teoría plantea la influencia de los factores ambientales y la necesidad de construir capacidades dinámicas para crear y responder a las circunstancias cambiantes del entorno (Lawson y Samson, 2001; Teece y Pisano, 1997). 
Entre las investigaciones realizadas que sugieren que determinadas contingencias incrementan o disminuyen la fuerza de la relación entre el mercado y el desempeño de las organizaciones está la de Kohli y Jaworsky (1990). Estos autores proponen como variables moderadoras de esta relación la turbulencia del mercado, la turbulencia tecnológica y la intensidad competitiva. La turbulencia de mercado es considerada como la medida en la cual los gustos y preferencias de los clientes son considerados. En su opinión, es mayor la probabilidad de que organizaciones que operan en mercados turbulentos tiendan a modificar sus productos y servicios continuamente para satisfacer las preferencias cambiantes de sus clientes. Por el contrario, las organizaciones en ambientes estables requerirán pocas modificaciones en sus productos. Asimismo, señalan que en ausencia de competencia las organizaciones tendrán un buen desempeño, aun si éstas no están orientadas al mercado. Sin embargo, bajo condiciones de alta competencia, los clientes tendrán diversas opciones para satisfacer sus necesidades y deseos, razón por la cual las organizaciones no orientadas al mercado perderán muchos clientes. También refieren que las organizaciones que trabajan con tecnología de punta y que están experimentando cambios rápidos pueden ser capaces de obtener ventajas competitivas a través de la innovación tecnológica, en contraste con las que emplean tecnologías estables, que estarán pobremente posicionadas (Jaworski y Kohli, 1993).

Respecto al desarrollo de recursos y capacidades, la teoría basada en los recursos sugiere que las diferencias entre el desempeño de las firmas son producto de diferencias en los recursos, al igual que de variaciones en cómo éstos se convierten en capacidades y se aplican (Barney, 1986; Grant, 1991). Esta teoría enfatiza la importancia de los recursos internos y las capacidades de la firma en el contexto del ambiente competitivo y el desarrollo de recursos y capacidades organizacionales (Wernerfelt, 1984) que ayudan a la organización a manejar su ambiente y a incrementar su desempeño (Day, 1994). Por supuesto, no todos son recursos estratégicos: algunos de ellos pueden hacer que la estrategia no se lleve a cabo o se implemente, otros pueden llevar a la firma a crear e implementar estrategias que reduzcan su eficiencia y efectividad, unos más podrían no tener ningún impacto en el proceso estratégico. Sin embargo, la ventaja competitiva se deriva de los recursos y capacidades que una firma controla, que son valiosos, raros, imperfectamente imitables y no sustituibles (Barney, 1991; Wernerfelt, 1984).

Las fuerzas de mercado parecen tener un efecto significativo en la generación de recursos y capacidades organizacionales (Ynzunza et al., 2008), por lo que la tarea clave para la firma es identificar aquellas capacidades que provean una fuerte ven- 
taja competitiva (Sarbo et al., 2003), ya que éstas son las que permiten a una organización adaptarse a los cambios del medio ambiente en general y generar nuevas estrategias que crean valor (Eisenhardt y Martin, 2000); es decir, la capacidad de una organización para enfrentar un ambiente de cambios dinámicos y crear nuevos recursos, renovarlos o alterar su mezcla (Teece et al., 1997).

Para abordar los recursos y capacidades bajo este enfoque, los investigadores se han apoyado en la teoría de la contingencia, la cual señala que el desempeño organizacional (ventaja competitiva) es resultado de la alineación apropiada de las variables de diseño organizacional endógenas con las variables del contexto exógeno. En opinión de Ginsberg y Venkatraman (1985), el enfoque de contingencia de estrategia sugiere que para un cierto conjunto de condiciones organizacionales y ambientales existe una estrategia óptima. Por su parte, Aragón y Sharma (2003) consideran que diferentes niveles de variación ambiental requerirán diferentes grados de formalidad estratégica, como medios para ajustar los recursos organizacionales con las oportunidades y amenazas del ambiente general de los negocios. Ynzunza e Izar (2010) proponen también la variación de niveles de capacidades en función del dinamismo de mercado, de modo que permitan a las organizaciones adaptarse a los cambios del ambiente.

En la literatura reciente, varias capacidades han sido identificadas por ser particularmente críticas para un desempeño mejorado y exitoso. Las capacidades de relación con el mercado y la mercadotecnia ayudan a explorar las oportunidades de mercado y están relacionadas con un incremento en el desempeño y la habilidad de sostener la ventaja competitiva (Conan et al., 1990; Jaworski y Kohli, 1993). Day (1994) sugiere como capacidades los procesos de adentro hacia afuera — activados por los requerimientos del mercado, los desafíos competitivos y las oportunidades externas-, las capacidades de conexión necesarias para integrar los aspectos internos y externos, así como el desarrollo de la estrategia y nuevos productos. En este sentido, Sinkovics y Roath (2004) plantean la alineación de la estrategia con la flexibilidad operacional en firmas que siguen una estrategia orientada al competidor debido a que hay una relación significativa directa entre la orientación al competidor y el desempeño logístico.

Algunos investigadores han considerado como determinantes de la competitividad de los negocios los siguientes: el capital tecnológico e innovación, los recursos humanos y el diseño de la organización interna (Barney, 1991). Otros mencionan la innovación continua y el aprendizaje organizacional, siempre y cuando estén 
asociados con una estrategia ambiental proactiva, en virtud de que los patrones de capacidades efectivas varían con el dinamismo del mercado o el ambiente competitivo de los negocios (Aragón y Sharma, 2003). Lerner y Almor (2002) señalan que para construir las fortalezas del negocio y desarrollar las habilidades estratégicas, que finalmente forman la base de la ventaja competitiva, una firma necesita enfocarse en la planeación, entendida ésta como una capacidad estratégica que permite a las empresas desarrollar otras capacidades necesarias para lograr un alto desempeño. Otros refieren la influencia de las capacidades tecnológicas en la ventaja competitiva. Las capacidades tecnológicas consideradas como información y habilidades - técnicas, organizacionales e institucionales que permiten a las empresas productivas utilizar equipo e información eficientemente, incluyendo las capacidades de inversión, de producción en tecnología de procesos y producto, y las de vinculación (Lall, 1992) — son procesos que evalúan la capacidad de la firma de buscar las nuevas tecnologías, la innovación, así como la investigación y desarrollo (Vongpanitlerd, 1992).

Ynzunza e Izar (2010) proponen también la orientación a mercado como un medio para construir algún tipo de ventaja competitiva que ayude a minimizar el impacto de las fuerzas de mercado sobre los objetivos organizacionales y un mecanismo para alcanzar un desempeño superior. Asimismo, Miles y Snow (1978) consideran que, dependiendo de la orientación estratégica adoptada, la firma puede enfatizar algunos aspectos de la administración, tales como la posición tecnológica, innovación, diseño organizacional y la administración de los recursos humanos. Concluyen que estos aspectos de la administración pueden en gran medida determinar el desempeño y la eficiencia de un negocio.

Por último, el desempeño organizacional es un constructo en la investigación de negocios que siempre ha probado ser problemático. El retorno sobre la inversión, el volumen de ventas y la participación de mercado han sido los indicadores de desempeño más ampliamente utilizados porque son fáciles de comparar entre firmas de diferente tamaño, aun cuando operen en mercados diferentes y usen estándares contables distintos. Algunos de estos criterios fueron evaluados en relación con los competidores principales y estiman que la medida de rentabilidad (ROI) está en función de la estrategia competitiva de negocio seleccionada (Fahy et al., 2000; Vorhies y Morgan, 2003; Matsuno y Mentzer, 2000). Otros incluyen también aspectos para medir la ventaja competitiva, como la lealtad al cliente, el éxito de nuevos productos y la participación de mercado (Narver y Slater, 1990); asimismo, consideran como capacidades nucleares el servicio al cliente, la cali- 
dad y la innovación (Slater y Narver, 1994). Sin embargo, las nuevas estrategias y realidades competitivas sugieren apoyarse en otras variables para evaluar este constructo, como son la calidad, la efectividad de la manufactura, la innovación y la satisfacción del cliente. Se sabe que todas las medidas tienen sus propias fortalezas y debilidades y que los esfuerzos de medida deben ser apropiados a la estrategia elegida. Venkatraman y Ramaujan (1986) visualizan el desempeño como un constructo multidimensional con tres niveles, que contemplan aspectos financieros relacionados con el mercado, la calidad y la responsabilidad social.

Por otro lado, Kaplan y Norton (1996; 2001) complementan las medidas tradicionales de desempeño con las relacionadas con los clientes, los procesos internos, el aprendizaje y el crecimiento del personal. También sugieren enfocarse en las estrategias que crean valor mediante la administración de activos intangibles, incluyendo relaciones con los clientes, productos y servicios innovadores, procesos de operación y alta calidad, capacidades y conocimientos de la fuerza de trabajo, la tecnología de la información y los vínculos con clientes y proveedores, además del clima organizacional que alienta la innovación, la solución de problemas y el mejoramiento.

Paralelamente, Croteau y Bergeron (2001) mencionan que, dado que no existe una medida universal reconocida para este concepto, el desempeño organizacional puede ser evaluado usando datos objetivos o subjetivos. El enfoque objetivo se refiere a los datos financieros proporcionados por la organización, mientras que las medidas subjetivas se enfocan a captar la percepción del encuestado. Ynzunza e Izar (2010) consideran elementos de adaptabilidad, eficiencia operativa, rentabilidad, satisfacción del cliente y, por supuesto, el crecimiento organizacional. Ravichandran y Lertwongsatien (2005) también emplean dimensiones operativas y de mercado. Olson y colaboradores (2005) sugieren que el desempeño debería ser visto desde el contexto de los objetivos de la firma, la estrategia y la estructura de mercado; es decir, el desempeño total de la firma, debido a su relevancia, ya que esta medida influye no solamente en el desempeño de la organización y en las dimensiones de retorno sobre los activos, ventas y crecimiento, sino también en otros objetivos financieros y no financieros que pudieran ser importantes para las organizaciones. Finalmente, el desempeño financiero es un fenómeno de múltiples aspectos, difícil de medir, en el cual la investigación académica ha mostrado que tanto los indicadores cuantitativos como los cualitativos tienen ciertas limitaciones, por lo que se ha recomendado el uso de factores de ambos tipos. 


\section{Metodología}

La investigación es cualitativa y cuantitativa, considerada de tipo transversal, causal y tiene como unidad de análisis a la pequeña y mediana empresa del sector industrial del estado de Querétaro. La parte cualitativa se hizo mediante la técnica de grupo focal con una guía de preguntas estructuradas basada en el sustento teórico revisado con el fin de explorar el tema de estudio, determinar las dimensiones, subdimensiones y variables estratégicas de cada constructo y evaluar los ítems que podrían integrar los mismos; en éste participaron académicos y empresarios. Para la investigación cuantitativa se revisaron primeramente las escalas de medición empleadas en estudios anteriores; se seleccionaron los referentes métricos apropiados, se adecuaron al contexto y se construyeron otros que no habían sido generados previamente como los de tecnología e innovación y desempeño organizacional, que forman parte del cuestionario diseñado para llevar a cabo la investigación. En el caso de escalas nuevas, se recurrió para su formulación al procedimiento seguido por Jaworski y Kohli (1993). Este mismo panel participó en la revisión y delimitación del instrumento de medición final, señalando inconsistencias o confusión en la comprensión de algunos ítems, así como en la recomendación de incluir o eliminar algunos de ellos, iniciando así el proceso de validación.

Para la medición de todos los constructos, se hizo uso de una escala Likert, tipo ordinal de cinco puntos medida con un rango de "totalmente en desacuerdo" a "totalmente de acuerdo" respecto a la estrategia competitiva, las fuerzas de mercado y los recursos y capacidades orientados a mercado. El desempeño organizacional se evaluó con un rango de "mucho peor" a "mucho mejor que los competidores" respecto a los últimos tres años. Por último, y debido a que la literatura cita el efecto que variables como el tipo, tamaño de organización y sector económico pudiesen tener sobre los constructos estudiados, se incluyeron ítems que brindaran información descriptiva y de valor sobre estas variables. Concluido el instrumento de medición, se realizaron diversas pruebas de la validez y fiabilidad del mismo a través de la correlación de Pearson, alfa de Cronbach y análisis factorial y se procedió a su aplicación.

\section{Características de la muestra y la unidad muestral}

El muestreo que se llevó a cabo es de tipo no probabilístico, de conveniencia con base en la disposición de las organizaciones productivas a participar en la investigación. El método de muestreo fue mediante entrevista personalizada del inves- 
tigador con los ejecutivos preferentemente del área de dirección. El criterio de selección de empresas fue el número de empleados, el cual según la Secretaría de Economía es un referente válido de clasificación para definir el tamaño de las organizaciones. Esto delimitó el tamaño de la población a un total de 646 empresas. El tamaño de la muestra alcanzado fue de 116, lo que resulta adecuado para el modelo estadístico de ecuaciones estructurales planteado. En modelos de este tipo, Hair y colaboradores señalan como aceptable un tamaño mínimo de muestra de 100 a 150 casos. Las organizaciones que constituyeron la muestra de estudio estuvieron representadas en su mayoría por el sector metal mecánico, seguido del de química, caucho y plástico, alimentos, bebidas y tabacos, servicios industriales y en porcentajes menores algunas empresas de diferentes sectores minoritarios, como el eléctrico y electrónico, agroindustrial, madera, minerales no metálicos y otros. Las empresas encuestadas en su gran mayoría son medianas y pequeñas empresas, principalmente de capital extranjero o nacional y una cantidad mínima está constituida por inversión mixta. Respecto al tipo de conformación, más de la mitad son subsidiarias o forman parte de un corporativo y el resto está representado por sociedades independientes y empresas familiares, estas últimas en menor porcentaje. Con relación a los empresarios que proporcionaron la información, fueron primordialmente directores generales o dueños, gerentes de producción y administradores. Gran parte de los mismos tienen más de cinco años de antigüedad en la empresa y cuentan con experiencia en el área de entre seis y diez años (cuadros 1 y 2). 


\section{Cuadro 1}

\section{Características de la unidad muestral}

\begin{tabular}{clcc}
\hline Concepto & \multicolumn{1}{c}{ Clasificación } & Frecuencia & \% \\
\hline \multirow{5}{*}{ Sector } & Química, caucho y plástico & 27 & 23.3 \\
& Metal mecánica & 47 & 40.5 \\
& Alimentos, bebidas y tabacos & 12 & 10.3 \\
& Textil & 8 & 6.9 \\
& Servicios industriales & 10 & 8.6 \\
& Otros & 12 & 10.3 \\
\hline \multirow{2}{*}{ Tamaño } & Pequeña & 27 & 23.3 \\
& Mediana & 89 & 76.7 \\
\hline \multirow{2}{*}{ Origen del } & Nacional & 48 & 41.4 \\
capital & Extranjero & 62 & 53.4 \\
& Mixto & 6 & 5.2 \\
\hline \multirow{3}{*}{ Mercado } & Nacional & 38 & 32.8 \\
& Extranjero & 15 & 12.9 \\
& Mixto & 63 & 54.3 \\
\hline \multirow{3}{*}{ Tipo de empresa } & Familiar & 23 & 19.8 \\
& Sociedad independiente & 31 & 26.7 \\
& Subsidiaria y/o Coorporativo & 62 & 53.4 \\
\hline
\end{tabular}

Cuadro2

Características de los elementos muestrales

\begin{tabular}{clcc}
\hline Concepto & Clasificación & Frecuencia & \% \\
\hline \multirow{4}{*}{ Escolaridad } & Preparatoria & 13 & 11.2 \\
& Licenciatura & 81 & 69.8 \\
& Especialidad & 11 & 9.5 \\
& Maestría & 5 & 4.3 \\
& Otros & 6 & 5.2 \\
\hline \multirow{3}{*}{ Experiencia } & 1 a 5 años & 32 & 27.6 \\
& 6 a 10 años & 27 & 23.3 \\
& Más de 10 años & 57 & 49.1 \\
\hline \multirow{2}{*}{ Antigüedad en la } & 1 a 5 años & 45 & 38.8 \\
empresa & 6 a 10 años & 43 & 37.1 \\
& 11 a 20 años & 22 & 19 \\
& Más de 20 años & 6 & 5.2 \\
\hline \multirow{2}{*}{ Área } & Dirección general & 22 & 19.0 \\
& Producción & 57 & 49.1 \\
& Administración & 13 & 11.2 \\
& Otras & 24 & 20.7 \\
\hline
\end{tabular}


Para la medición de los constructos, en este caso la estrategia competitiva, se empleó la tipología de Miles y Snow (1978), ya que presenta una plataforma teórica particularmente útil para analizar la forma en la cual las organizaciones interactúan con sus ambientes. El enfoque seleccionado fue el autodescriptivo donde los gerentes evalúan las estrategias de sus propias organizaciones haciendo uso de las descripciones de las actividades propuestas, las cuales son las mismas que aquéllas usadas por Segev (1987) con algunas modificaciones para ajustarla a la industria queretana. Los recursos y capacidades de orientación al mercado se evaluaron con la conceptualización y operacionalización de Narver y Slater (1990), la cual ha sido utilizada para negocios pequeños y se considera se adapta mejor a los objetivos de la investigación. Respecto a las capacidades tecnológicas y de innovación, dos de las aportaciones más aceptadas y relacionadas directamente con la investigación son la de Lall (1992) y Vongpanitlerd (1992); sin embargo, su enfoque ha sido teórico, por lo que se desarrollaron y validaron las escalas de medición para explorar las mismas. En relación con el ambiente en el cual operan las organizaciones, se tomaron los instrumentos desarrollados por Jaworski y Kohli (1993) considerando tres dimensiones, la turbulencia tecnológica, de mercado y la intensidad competitiva. Finalmente, de los diversos enfoques encontrados sobre desempeño organizacional, el de Venkatraman y Ramanujam (1986) ha sido de los más aceptados, al igual que el de Vorhies y Harker (2000), por lo que algunas de sus contribuciones se usaron para diseñar los ítems que formaron parte de esta contrucción teórica.

\section{Análisis y resultados}

Los resultados encontrados en las organizaciones de la muestra de estudio presentan información valiosa sobre la industria del estado. Las fuerzas de mercado tienen un impacto fuerte sobre las organizaciones; muchos de los empresarios encuestados consideran que las preferencias de sus clientes, al igual que la tecnología, cambian constantemente, lo que en ocasiones genera oportunidades para sus organizaciones y coinciden en que continuamente se ven afectados por nuevos movimientos competitivos. El mayor problema que enfrentan las organizaciones queretanas parece estar en los aspectos competitivos y de mercado, los cuales repercuten de manera distinta por tamaño, tipo de empresa y sector. En este caso, es manifiesto el predominio de las cuestiones competitivas tanto en pequeñas como en medianas empresas; sin embargo, la turbulencia tecnológica parece impactar más a las pequeñas empresas y los aspectos de mercado a las medianas, las familiares e independientes, y no así con las subsidiarias. 
Con referencia a la estrategia competitiva, las actividades con menores niveles de realización son las prospectoras; la mayoría de las organizaciones no considera que sus organizaciones dirijan las innovaciones en su industria, no desarrollan nuevos productos, ni nuevas actividades competitivas y dicen no tener la capacidad para adoptar rápidamente las innovaciones prometedoras. Los resultados también muestran que la empresa pequeña está limitada para llevar a cabo actividades prospectoras y analizadoras encontrándose en estas actividades los valores con los niveles más bajos. En contraste, las empresas medianas dan prioridad a actividades analizadoras, al igual que las subsidiarias e independientes. En cuanto a los recursos y capacidades organizacionales, los empresarios coinciden en que de alguna manera tienen o desarrollan en sus organizaciones los dos tipos de recursos y capacidades evaluados. Los mayores porcentajes se observan en las dimensiones de tecnología e innovación. Los hallazgos por tamaño coinciden aunque en menor medida en la pequeña empresa. Respecto al tipo de empresa se encuentran similitudes en el uso de capacidades de tecnología e innovación en las subsidiarias de corporativos y sociedades. El desempeño organizacional fue asociado con el crecimiento, aunque mayormente refieren situaciones de tipo operativo y de satisfacción al cliente. Esta misma tendencia se refleja en el tamaño de las organizaciones y en los aspectos operativos, aunque las pequeñas dan más énfasis al crecimiento y las medianas a la de satisfacción del cliente.

El análisis por sector muestra diferencias en cada uno de los constructos analizados. Respecto a las actividades estratégicas, el sector química, caucho y plástico favorece las actividades analizadoras sobre las prospectoras, al igual que el sector metal mecánica, el textil y otros minoritarios como el eléctrico, electrónico, agroindustrial, madera, minerales no metálicos, etc. Los porcentajes más altos referidos para actividades analizadoras se hallan en los sectores minoritarios, seguidos del de química, caucho y plástico, metalmecánica, textil y el mínimo en el de alimentos, bebidas y tabacos. En contraste, las prospectoras sobresalen en el sector de servicios industriales y el de alimentos, bebidas y tabacos, y otros minoritarios, en donde el textil es el que presenta los niveles más bajos. El efecto de las fuerzas de mercado es también distinto para cada sector: en el caso del metal mecánica y el de química, caucho y plástico no se ven afectados fuertemente por los cambios que se generen en el mercado, en cambio en el textil, en el de alimentos, bebidas y tabaco, y en el de servicios tecnológicos industriales el impacto se considera significativo. 
En cuanto a los aspectos tecnológicos impactan en menor medida los sectores metal mecánica, química, caucho y plásticos y el textil, y en mayor porcentaje el de alimentos, bebidas y tabacos y otros sectores minoritarios. Con referencia a la intensidad competitiva todos coinciden en que es un problema que enfrentan sus organizaciones, afectando principalmente al sector de alimentos, bebidas y tabacos, química, caucho y plástico, otros minoritarios y el de metal mecánica. Respecto al desempeño, son el de química, caucho y plástico y servicios tecnológicos, los que asignan una mayor importancia a los aspectos relacionados con el crecimiento organizacional. En relación con los recursos y capacidades, todos los sectores parecen privilegiar el desarrollo de recursos tecnológicos y de innovación sobre los de mercado. Sin embargo, existen diferencias en los porcentajes asignados, encontrándose los mayores en el sector de servicios tecnológicos industriales y el de alimentos, bebidas y tabacos, mientras que en el caso de los recursos de orientación al mercado se hallan en otros sectores minoritarios. El cuadro 3 concentra los porcentajes obtenidos con el mayor rango de respuesta por constructo en cada dimensión.

\section{Cuadro 3}

\section{Resultados por sector 0 actividad económica}

\begin{tabular}{|c|c|c|c|c|c|c|c|c|}
\hline \multirow{4}{*}{$\begin{array}{l}\text { Sector } \\
\text { Química, } \\
\text { caucho y } \\
\text { plástico }\end{array}$} & \multicolumn{2}{|c|}{ Fuerzas de mercado } & \multicolumn{2}{|c|}{$\begin{array}{r}\text { Estrategia } \\
\text { competitiva }\end{array}$} & \multicolumn{2}{|c|}{$\begin{array}{c}\text { Recursos y } \\
\text { capacidades }\end{array}$} & \multicolumn{2}{|c|}{$\begin{array}{c}\text { Desempeño } \\
\text { organizacional }\end{array}$} \\
\hline & FMTUME & 29.6 & ACTPRO & 37.0 & RCOMO & 44.4 & \multirow{3}{*}{ DOCRE } & \multirow{3}{*}{70.4} \\
\hline & FMTUTE & 37.0 & \multirow{2}{*}{ ACTANA } & \multirow{2}{*}{48.1} & \multirow{2}{*}{ RCOTEI } & \multirow{2}{*}{48.2} & & \\
\hline & FMINCO & 40.7 & & & & & & \\
\hline \multirow{3}{*}{$\begin{array}{l}\text { Metal } \\
\text { mecánica }\end{array}$} & FMTUME & $36.2^{*}$ & ACTPRO & 29.8 & RCOMO & 40.4 & \multirow{3}{*}{ DOCRE } & \multirow{3}{*}{38.3} \\
\hline & FMTUTE & 31.9 & \multirow{2}{*}{ ACTANA } & \multirow{2}{*}{38.3} & \multirow{2}{*}{ RCOTEI } & \multirow{2}{*}{55.3} & & \\
\hline & FMINCO & 40.4 & & & & & & \\
\hline \multirow{3}{*}{$\begin{array}{c}\text { Alimentos, } \\
\text { bebidas y } \\
\text { tabacos }\end{array}$} & FMTUME & 58.3 & $\mathrm{O}$ & 41.7 & RCOMO & 41.7 & \multirow{3}{*}{ DOCRE } & \multirow{3}{*}{50.0} \\
\hline & FMTUTE & 58.3 & \multirow{2}{*}{ ACTANA } & \multirow{2}{*}{33.3} & \multirow{2}{*}{ RCOTEI } & \multirow{2}{*}{66.7} & & \\
\hline & FMINCO & 50.0 & & & & & & \\
\hline \multirow{3}{*}{ Textil } & FMTUME & 62.5 & ACTPRO & 25.0 & RCOMO & 25.0 & \multirow{3}{*}{ DOCRE } & \multirow{3}{*}{50.0} \\
\hline & FMTUTE & $37.5^{*}$ & ACTANA & 375 & RCOTEI & 375 & & \\
\hline & FMINCO & 37.5 & & & & & & \\
\hline \multirow{3}{*}{$\begin{array}{l}\text { Servicios } \\
\text { industriales }\end{array}$} & FMTUME & 60.0 & ACTPRO & 70.0 & RCOMO & 50.0 & \multirow{3}{*}{ DOCRE } & \multirow{3}{*}{80.0} \\
\hline & FMTUTE & 40.0 & ACTANA & 400 & RCOTEI & 700 & & \\
\hline & FMINCO & 40.0 & 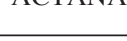 & & & & & \\
\hline \multirow{3}{*}{$\begin{array}{l}\text { Otros } \\
\text { sectores }\end{array}$} & FMTUME & 50.0 & ACTPRO & 41.7 & RCOMO & 50.0 & \multirow{3}{*}{ DOCRE } & \multirow{3}{*}{58.3} \\
\hline & FMTUTE & 50.0 & ACTANA & 66.7 & RCOTEI & 58.3 & & \\
\hline & FMINCO & $41.7 * *$ & & & & & & \\
\hline
\end{tabular}

Nota: En acuerdo * En desacuerdo ** Ni en acuerdo ni en desacuerdo 
Para probar las relaciones supuestas entre las variables dependientes e independientes, se utilizó la modelación estructural (SEM); para ello se construyó el modelo teórico y de medida. El modelo es de tipo recursivo, lo cual permite que los errores no estén correlacionados y que todos los efectos causales sean unidireccionales (Kline, 1998). Los pesos y cargas de los ítems reflejan la robustez de las medidas y el signo el sentido de las relaciones teóricas (Hair et al., 1999). El procesamiento de la información se hizo mediante el software SPSS y AMOS. La consistencia interna se midió a través del alfa de Cronbach. Los valores encontrados se muestran en el cuadro 4. La validez discriminante se probó mediante el análisis factorial confirmatorio con el método de componentes principales y rotación varimax. El valor del determinante alcanzado fue de 0.033 , la medida de adecuación de la muestra de Kaiser Meyer Olkin fue 0.794 y la prueba de esfericidad de Bartlett 381.027 con 28 grados de libertad. Las medidas de adecuación muestral (MSA) fueron de 0.714 la mínima y de 0.859 la máxima. El cuadro 5 muestra la estructura factorial del modelo desarrollado y la varianza total explicada de cada uno de los constructos. La evaluación del modelo señala que el ajuste mínimo fue alcanzado sin estimaciones infractoras o errores. Las medidas de calidad de ajuste del modelo reflejan una chi cuadrada de 27.995, con 15 grados de libertad y un nivel de significancia de 0.022. El resultado de la bondad de ajuste del modelo fue de 1.866. Los índices estadísticos obtenidos para evaluar el ajuste del modelo muestran un GFI de 0.949, un RMSEA de 0.087, un TLI de 0.937 y un RMR de 0.051. Las diversas medidas globales de la calidad de ajuste aportan resultados suficientes para considerar los hallazgos como una medida confiable de la representación de los constructos. El modelo de investigación diseñado y los resultados obtenidos se muestran en la figura 1.

\section{Cuadro 4 Alfa de Cronbach}

\begin{tabular}{cccc}
\hline \multirow{2}{*}{ Constructo } & Variables & Confiabilidad & No. \\
\cline { 3 - 3 } & Alfa de Cronbach & Ítems \\
\hline \multirow{3}{*}{ Fuerzas de mercado } & Turbulencia de mercado & 0.811 & 3 \\
& Turbulencia tecnológica & 0.910 & 3 \\
& Intensidad competitiva & 0.789 & 3 \\
\hline Estrategias competitivas & Prospectoras & 0.925 & 5 \\
orientadas a mercado & Analizadoras & 0.736 & 2 \\
\hline Recursos y capacidades & Tecnología e innovación & 0.950 & 17 \\
orientados a mercado & Orientación a mercado & 0.900 & 9 \\
\hline Desempeño organizacional & Crecimiento & 0.866 & 3 \\
\hline
\end{tabular}




\section{Cuadro 5}

\section{Análisis factorial confirmatorio de los constructos del modelo}

\begin{tabular}{ccccc}
\hline Variables & $\begin{array}{c}\text { Fuerzas de } \\
\text { mercado }\end{array}$ & $\begin{array}{c}\text { Estrategia } \\
\text { competitiva }\end{array}$ & $\begin{array}{c}\text { Recursos y } \\
\text { capacidades }\end{array}$ & $\begin{array}{c}\text { Desempeño } \\
\text { organizacional }\end{array}$ \\
\hline FMTUME & .127 & .029 & $\mathbf{. 8 9 6}$ & .245 \\
FMTUTE & .518 & .190 & $\mathbf{. 6 1 2}$ & .144 \\
FMINCO & .253 & .404 & $\mathbf{. 6 7 7}$ & -.323 \\
RCOMO & .266 & $\mathbf{. 8 4 0}$ & .115 & .242 \\
RCOTEI & .186 & $\mathbf{. 8 4 5}$ & .153 & .130 \\
ESTPRO & $\mathbf{. 8 8 4}$ & .150 & .212 & .131 \\
ESTANA & $\mathbf{. 7 8 6}$ & .349 & .177 & .127 \\
DOCRE & .238 & .329 & .145 &. $\mathbf{8 4 3}$ \\
\hline Varianza & 23.868 & 23.411 & 22.145 & 12.545 \\
\hline Var. Acumulada & 23.868 & 47.278 & 69.423 & 81.968 \\
\hline
\end{tabular}

Método de extracción: Análisis de componentes principales Método de rotación: Varimax con normalización Kaiser Rotación convergió en seis interacciones

Figura 1

Modelo estructural desarrollado

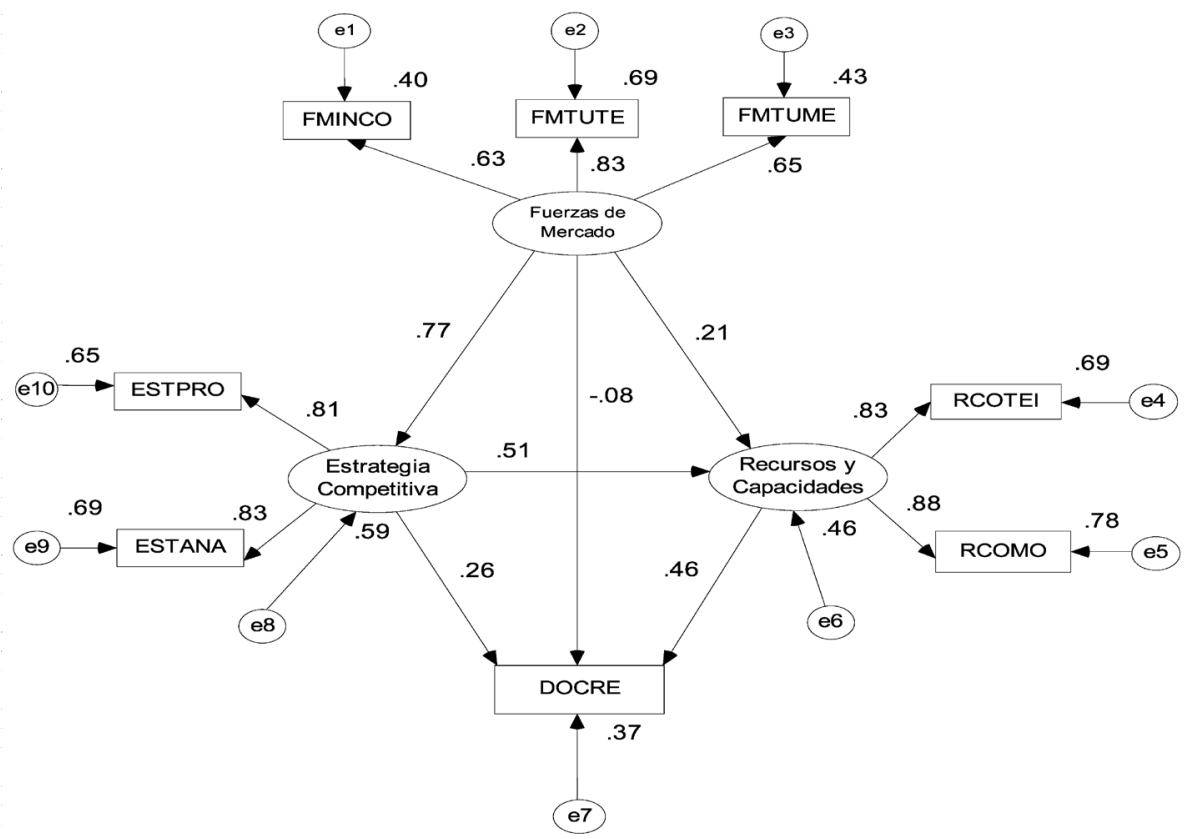




\section{Discusión}

La primera relación planteada en la investigación busca conocer el efecto de las fuerzas de mercado sobre las estrategias competitivas orientadas a mercado. Los resultados muestran que la relación es significativa y positiva con un efecto considerado grande $(\beta .77 ; \mathrm{p}<.001)$. Las dimensiones que forman parte de las fuerzas de mercado también fueron todas significativas con efectos considerables: la turbulencia de mercado $(\beta .65 ; \mathrm{p}<.001)$, la turbulencia tecnológica $(\beta .83 ; \mathrm{p}<.001)$ y la intensidad competitiva $(\beta .63 ; \mathrm{p}<.001)$. Los hallazgos obtenidos sustentan la teoría existente respecto a la asociación de este constructo con la estrategia competitiva. Los cambios del entorno, producto de la turbulencia de mercado, tecnológica o la intensidad competitiva, tienen un efecto significativo en las actividades estratégicas, favoreciendo o inhibiendo su interacción y adaptación al medio ambiente. Esta interacción parece ser producto de las actividades estratégicas implementadas (Matsuno y Mentzer, 2000), en este caso prospectoras y analizadoras, y podría ser un precedente del éxito organizacional (Mc Daniel y Kolari, 1987).

Sin embargo, se encuentra que otras variables como el tamaño, el tipo de organización y el sector económico modifican también la influencia de éstas; por tanto, las propias condiciones de las organizaciones y el efecto que los cambios ambientales pudieran tener en las mismas requerirán la implementación de diferentes tipos y niveles de actividades estratégicas como medio para acoplar sus recursos y capacidades organizacionales al ambiente general de los negocios (Aragón y Sharma, 2003). El segundo supuesto planteado explora la relación existente entre las fuerzas de mercado y el desarrollo de recursos y capacidades orientados a mercado; los hallazgos señalan que ésta es positiva, pero no significativa $(\beta .21 ; \mathrm{p}<.05)$. De igual manera, los estimados para las dimensiones que conforman los recursos y capacidades fueron positivos y significativos con efectos superiores: los recursos y capacidades de tecnología e innovación $(\beta .83 ; \mathrm{p}<.001)$ y los de orientación a mercado $(\beta .88 ; \mathrm{p}<.001)$.

Los resultados alcanzados confirman que los aspectos de mercado, competitivos y tecnológicos por sí solos no propician el desarrollo de recursos y capacidades. Es a través de la implementación de estrategias competitivas orientadas a mercado - como las prospectoras y analizadoras - como se potencializa la creación de estos recursos. Asimismo, los recursos y capacidades de tecnología e innovación al igual que los de orientación a mercado son determinantes de la competitividad de los negocios (Barney, 1991; y Eisenhardt y Martin, 2000) y están efectivamente 
vinculados con el crecimiento organizacional. Las capacidades técnicas ayudan a mejorar la eficiencia productiva, reducir costos e incrementar su competitividad; las de orientación a mercado ayudan a explorar las oportunidades, ambas están relacionadas con un incremento en el desempeño y la habilidad de sostener la ventaja competitiva (Conan et al., 1990). No obstante, la orientación a mercado parece tener un mayor peso en el logro de los objetivos organizacionales. La tercera hipótesis vincula las fuerzas de mercado con el desempeño medido por el crecimiento organizacional; los resultados refieren que la relación es negativa y no significativa $(\beta-.08 ; \mathrm{p}<.05)$. Lo anterior refleja el impacto negativo que tienen las fuerzas de mercado en el desempeño organizacional.

Las condiciones del entorno alteran la relación entre el mercado y las organizaciones; es por ello que es importante conectar los procesos organizacionales con el ambiente externo para anticiparse a los movimientos competitivos, tecnológicos y de mercado, dado que factores como la turbulencia, la demanda y la competencia de mercado definirán el nivel de innovación, tecnología y orientación a mercado que requieren las organizaciones para posicionarse y alcanzar un mejor desempeño (Kohli y Jaworsky, 1990). El cuarto supuesto investiga la relación existente entre la implementación de estrategias competitivas y el desarrollo de recursos y capacidades orientados a mercado. El vínculo es positivo y significativo con un efecto considerado como elevado $(\beta .51 ; \mathrm{p}<.01)$. Las dimensiones que representan a la estrategia competitiva también son significativas con efectos grandes para las estrategias competitivas prospectoras $(\beta .81 ; \mathrm{p}<.001)$ y las analizadoras $(\beta .83 ; \mathrm{p}<.001)$. A este respecto, los hallazgos obtenidos muestran que las estrategias competitivas prospectoras y analizadoras promueven el desarrollo de organizaciones orientadas al mercado, la tecnología y la innovación.

Los hallazgos encontrados prueban que empresas con mayores niveles de actividades estratégicas prospectoras y analizadoras favorecen el desarrollo de recursos y capacidades organizacionales relacionados con la tecnología e innovación y orientación a mercado, asociados significativamente con el desempeño organizacional (Day, 1994). Las implicaciones implícitas son que, dependiendo de la orientación estratégica adoptada, la firma puede enfatizar o no cierto tipo de aspectos, lo que en gran medida podría determinar el desempeño y la eficiencia de negocio. La fuerte asociación entre los tipos estratégicos y las capacidades de la firma evidencia que las empresas prospectoras, por ejemplo, utilizan tanto las estrategias de mercado como las de tecnología e innovación para anticiparse a nuevos productos y oportunidades (Miles y Snow, 1978). Sin embargo, su eficiencia parece ser contingente 
también a otros factores como el tamaño, tipo de empresa y sector industrial. En términos de recursos estratégicos algunas firmas en una industria podrían ser idénticas, pero los recursos para implementar sus estrategias cambian constantemente (Barney, 1991; Wernerfelt, 1984), por lo que podría afectarse su implementación, eficiencia y efectividad (Barney, 1986).

El siguiente supuesto explora la asociación entre el desarrollo de recursos y capacidades orientados a mercado con el desempeño. El vínculo encontrado es positivo y significativo $(\beta .46 ; \mathrm{p}<.001)$, lo cual refleja la importancia que tiene para el éxito de las organizaciones el desarrollo de recursos y capacidades orientados al mercado. Los resultados obtenidos muestran que tanto los recursos y capacidades orientados a mercado como los de innovación y tecnología están vinculados al desempeño. Esto es de enorme trascendencia, ya que los recursos y capacidades organizacionales que la organización posea y desarrolle tendrán un impacto significativo en el desempeño de las organizaciones, a la par de la estrategia competitiva seleccionada. En términos prácticos significa que en la medida en que una organización fortalezca y amplíe sus prácticas de orientación al mercado y de tecnología e innovación estará en mejores posibilidades de alcanzar un desempeño superior al de sus competidores principales, caracterizando así a organizaciones prospectoras y analizadoras. De esta forma, algunas capacidades, como las de relación con el mercado y desarrollo tecnológico y la innovación podrían ser más críticas para un éxito continuado (Conan et al., 1990).

La última hipótesis plantea el efecto de la implementación de estrategias competitivas orientadas a mercado con el desempeño organizacional. El efecto es positivo, pero no significativo con un peso considerado grande $(\beta .26 ; \mathrm{p}<.05)$. Los resultados de este supuesto corroboran que el impacto de la estrategia competitiva está en la capacidad de generar recursos y capacidades organizacionales orientados a mercado; por sí misma la estrategia competitiva no garantiza el crecimiento de las organizaciones. El vínculo entre los tipos estratégicos y el desempeño no es tan directo como se sugiere (Shortell y Zajac, 1990), aun cuando estrategias prospectoras y analizadoras han sido referidas al mismo. Variables como el tamaño, los atributos ambientales y la alineación entre la estrategia y los recursos parecen afectar estos resultados. La premisa básica aquí es la necesidad de que las organizaciones tengan una mayor consistencia entre la estrategia, el modelo y tipo de negocio y sus capacidades organizacionales. 


\section{Conclusiones}

Los resultados de la investigación responden a las preguntas de investigación planteadas. La relación existente entre las fuerzas de mercado y la implementación de estrategias competitivas orientadas a mercado fue positiva, probando así el impacto que tienen las fuerzas de mercado, evaluadas por la turbulencia de mercado, la turbulencia tecnológica y la intensidad competitiva en la determinación de la estrategia competitiva y validando la importancia de adoptar estrategias prospectoras y analizadoras, las cuales permiten una mejor adaptación a los cambios que se gestan en el entorno e incentivan los aspectos que contribuyen a generar crecimiento, coincidiendo con los hallazgos de Hambrick (1983), Miles y Snow (1978) y Aragon y Sharma (2003) sobre la influencia del ambiente en la efectividad de la estrategia competitiva. No así respecto al vínculo existente entre las fuerzas de mercado y el desarrollo de recursos y capacidades orientados a mercado, cuyos resultados fueron no significativos. De igual manera, no se probó el efecto positivo de las fuerzas de mercado sobre el crecimiento de las organizaciones, sino más bien se halló que éste es negativo, aunque no significativo.

En cuanto a la implementación de estrategias prospectoras y analizadoras, el desarrollo de recursos y capacidades orientados a mercado y el vínculo de estos últimos con el crecimiento organizacional, los hallazgos prueban que la asociación es positiva y significativa, enfatizando así la importancia de generar capacidades estratégicas como las de tecnología e innovación y de orientación a mercado para el desempeño futuro de las organizaciones, que dependen en gran medida de la implementación de estrategias competitivas que propicien el desarrollo de las mismas, comprobando así los hallazgos de Narver y Slater (1990), Jaworski y Kohli (1993) y Conan et al. (1990). Finalmente, la relación entre la adopción de estrategias competitivas orientadas a mercado y el crecimiento de las organizaciones fue corroborada, aunque su impacto no es significativo. Por ello, puede concluirse que la selección de estrategias competitivas orientadas a mercado, así como las prospectoras y analizadoras, favorecen la generación de recursos y capacidades que podrían ser pilares en la construcción de algún tipo de ventaja competitiva, ya que ambas contribuyen a maximizar los objetivos organizacionales y permiten hacer frente a las condiciones adversas que se gestan en un entorno altamente dinámico y competitivo, en el cual están inmersas las empresas mexicanas. Sin embargo, la estrategia competitiva por sí sola no contribuye al crecimiento organizacional, sino que a través de la generación de los recursos y capacidades distintivas y estratégicas es como las organizaciones podrían alcanzar un crecimiento y desempeño sos- 
tenido en el largo plazo. La correcta alineación de la estrategia competitiva con las fuerzas de mercado es lo que favorecerá o limitará el desarrollo de estos recursos, por lo cual la necesidad de que las organizaciones cuenten con mecanismos que les permitan monitorear las condiciones competitivas, tecnológicas y de mercado, lo que facilitará su adaptación y garantizará la satisfacción de las necesidades y expectativas de sus clientes. No obstante, esto depende también en gran medida del tamaño, tipo de organización y el sector económico en el cual estén inmersas las organizaciones.

\section{Propuesta para futuros estudios}

Distintas líneas de investigación pueden sugerirse con base en los resultados de este estudio. En primer lugar, la investigación contempla únicamente el desarrollo de recursos de tecnología e innovación; sin embargo, no considera la importancia de generar otros recursos y capacidades para crear los mismos, como la gestión del conocimiento y el liderazgo. En segundo término, el desempeño fue medido únicamente con medidas de percepción; podrían emplearse otras más objetivas como los indicadores de rentabilidad y crecimiento en relación con industrias similares. Por último, el estudio se llevó a cabo solamente en el estado de Querétaro; éste podría replicarse en otros estados.

\section{Bibliografía}

Aragón, J. y S. Sharma (2003). A Contingent Resource Based View of Proactive Corporate Environmental Strategy. Academy of Management Review 28 (1): 71-88.

Barney, J. (1986). Strategic factors markets, expectations, luck, and business strategy. Management Science 42 (10): 1231-1241.

(1991). Firm Resources and Sustained Competitive Advantage. Journal of Management 17 (1): 99-10.

Camisón, C. (1997). La Competitividad de la PYME Industrial Española: Estrategia y Competencias Distintivas, Madrid: Civitas. 
Conan, J., M. Mokwa, y P. Varadarajan (1990). Strategic types, distinctive marketing competences and organizational performance: A multiple-measuresbased study. Strategic Management Journal 11 (5): 365-383.

Croteau, A. y F. Bergeron (2001). An information technology trilogy: business strategy, technological deployment and organizational performance. Journal of Strategic Information Systems 10 (2): 77-99.

Day, G. (1994). The Capabilities of Market Driven Organizations. Journal of Marketing 58 (4): 37-52.

De Sarbo, W., A. Di Benedetto, K. Jedidi and M. Song (2003). A Constrained Finited Mixture Structural Equation Methodology for Empirically Deriving Strategic Types. Institute for the Study of Business Markets, Pennsylvania State University.

Díaz, F. (2002), Reseña de Claroscuros. Integración exitosa de las Pequeñas y Medianas Empresas en México, Revista de la Facultad de Economía (19): 181-184.

Eisenhardt, K. y J. Martin (2000). Dynamic Capabilities: What are they? Strategic Management Journal 21 (10/11): 1105-1121.

Fahy, J., G. Hooley, T. Cox, J. Beracs, K. Fonfara y B. Snoj (2000). The Development and Impact of Marketing Capabilities in Central Europe. Journal of International Business Studies 31 (1): 63-81.

Freeman, C., J. Clark y L. Soete (1982). Unemployment and Technical innovation, London: Pinter.

Ginsberg, A. y N. Venkatraman (1985). Contingency Perspective of Organizational Strategy: A critical Review of the Empirical Research. Academy of Management Review 10 (3): 421-434.

Grant, R. (1991). The resource based theory of competitive advantage: Implications for strategy formulation. California Management Review 33 (3): 114135. 
Hair, J., R. Anderson, R. Tatham y W. Black (1999). Análisis Multivariante, 5a. edición, Madrid: Pearson Prentice Hall.

Hambrick, D. (1983). Some Tests of the effectiveness and functional attributes of Miles y Snow's Strategic Types. Academy of Management Journal 26 (1): $5-26$.

Instituto Nacional de Estadística, Geografía e Informática (2004). Micro, Pequeña, Mediana y Gran Empresa. Estratificación de los Establecimientos. Censos Económicos.

Jaworski, B. y A. Kohli (1993). Market Orientation: Antecedents and Consequences. Journal of Marketing 57 (3): 53-70.

Kaplan, R. y D. Norton (1996). Using the Balanced Scorecard as a Strategic Management System. Harvard Business Review: 75-85.

(2001). Transforming the Balanced Scorecard from Performance Measurement to Strategic Management: Part I, American Accounting Association. Accounting Horizons 15 (1): 87-104.

Kauffman, S. (2001). El Desarrollo de la Micro, Pequeñas y Medianas Empresas. Un Reto para la Economía Mexicana. Revista Ciencia Administrativa (1): 40-48.

Kline, R. (1998). Principles and practice of structural equation modeling, New York: Guilford Press.

Kohli, A. y B. Jaworski (1990). Market Orientation: The Construct, Research Propositions, and Managerial Implications. Journal of Marketing 54 (2): 1-18.

Lall, S. (1992). Technological Capabilities and Industrialization. World Development 20 (2): 165-186.

Lawson, B. y D. Samson (2001). Developing Innovation Capability in organizations: A Dynamic Capabilities Approach. International Journal of Innovation Management 5 (3): 378-400. 
Lerner, M. y T. Almor (2002). Relationships among Strategic Capabilities and the Performance of Women-Owned Small Ventures. Journal of Small Business Management 40 (2): 109-125.

Matsuno, K. y J. Mentzer (2000). The Effects of Strategy Type on the Market Orientation - Performance Relationship. Journal of Marketing 64 (4): 1-16.

McDaniel, S. y J. Kolari (1987). Marketing Strategy Implications of the Miles y Snow Strategic Typology. Journal of Marketing 52 (4): 19-30.

Miles, R. y C. Snow (1978). Organizational Strategy, Structure, and Process, New York: Mc Graw Hill.

Narver, J. y F. Slater (1990). The Effect of a Market Orientation on Business Profitability. Journal of Marketing 54 (4): 20-35.

Olson, E., S. Slater y T. Hult (2005). The Performance Implications of Fit Among Business Strategy, Marketing Organization Structure, and Strategic Behavior. Journal of Marketing, 69 (3): 49-65.

Porter, M. (1980). Competitive Strategy: Techniques for analyzing Industries and Competitors, New York: Free Press.

Ravichandran, T. y Ch. Lertwongsatien (2005). Effect of Information Systems Resources and Capabilities on Firm Performance: A Resourced Based Perspective. Journal of Management Information Systems 21 (4): 237-276.

Secretaría de Desarrollo Sustentable del Estado de Querétaro (2006). Informe Anual de Gobierno.

Segev, E. (1987). Strategy, Strategy Making and Performance - An empirical investigation. Management Science 33 (3): 258-269.

Sinkovics, R. y A. Roath (2004). Strategic Orientation, Capabilities and Performance in Manufacturer - 3PL Relationships. Journal of Business Logistics 25 (2): 43-64. 
Slater, S. y J. Narver (1994a). Market Orientation, Customer Value, and Superior Performance. Business Horizons 37 (22): 22-28.

(1994b). Does competitive environment moderate the market orientation-performance relationship? Journal of Marketing 58 (1): 46-55.

Teece, D., G. Pisano y S. Shuen (1997). Dynamic Capabilities and Strategic Management. Strategic Management Journal 18 (7): 509-533.

Venkatraman, N. (1989). Strategic orientation of business enterprises: The construct, dimensionality and measurement. Management Science 35 (8): 942962.

- y V. Ramanujam (1986). Measurement of business performance in strategy research: a comparison of approaches. Academy of Management Review 11 (4): 801-814.

Vongpanitlerd, S. (1992). The Development of Thailand's Technological Capability in Industry. Research Monograph, Thailand Development Research Institute (9): 121-152.

Vorhies, D., y M. Harker (2000). The Capabilities and Performance Advantages of Market Driven Firms: An Empirical Investigation. Australian Journal of Management 25 (2): 145-171.

y N. Morgan (2003). A Configuration Theory Assessment of Marketing Organization Fit with Business Strategy and Its Relationship with Marketing Performance. Journal of Marketing 67 (1): 100-115.

(2005). Benchmarking Marketing Capabilities for Sustainable Competitive Advantage. Journal of Marketing, 69 (1): 80-94.

Wernerfelt, B. (1984). A resource based view of the firm. Strategic Management Journal 5 (2): 171-180.

Ynzunza, C., A. Márquez y J. Izar (2008). El Efecto de las Fuerzas de Mercado en la Estrategia Competitiva, los Recursos y Capacidades Organizacionales y el Desempeño. Hitos de ciencias Económico Administrativas 14 (39): 55-64. 
y J. Izar (2010). Impacto de las Fuerzas de Mercado y la Orientación a Mercado en el Desempeño Organizacional, RIA 106 (39): 7-16.

(2010). Modelo de Estrategia Competitiva desde la Perspectiva de los Recursos y las Capacidades Organizacionales. Estudio del Sector Industrial del Estado de Querétaro. De la Educación a la Gestión Organizacional. Enfoques Diversos, Instituto Tecnológico de Aguascalientes. 
